\title{
La hegemonía y la integración económica regional en el noreste asiático
}

Nam Kwon Mun*

$\mathrm{P}$ odemos apreciar en la actualidad que la mayor parte de los países mantienen vínculos económicos con el mundo a través de bloques comerciales. Si se incluye al APEC (Foro de Cooperación Económica AsiaPacífico) como tratado comercial, ${ }^{1}$ prácticamente todos los países pertenecerían al menos a una de estas organizaciones.

Sin embargo, a diferencia de las otras regiones, la parte del noreste asiático ha estado distanciada de las olas de regionalismo hasta hace poco. ${ }^{2}$ Esto se debió a varios factores que siguen vigentes todavía. Pero ahora se aprecia la voluntad y el movimiento de los países de esta región para establecer los tratados de libre comercio que cambiarán el panorama económico. Este nuevo movimiento de regionalismo en el noreste asiático se concreta actualmente como una parte de la estrategia para conseguir la supremacía tanto política como económica de la región. Este artículo analiza el proceso de la formación de los TLC y su relación con la lucha por la hegemonía entre Japón y China en el noreste asiático.

\section{Regionalización y regionalismo}

La integración económica regional representada por los tratados de libre comercio se puede definir como un regionalismo. El regionalismo es una modalidad políticamente desarrollada de la regionalización. La regionalización es actualmente una tendencia universal que afecta a todos los países por la economía mundial. ${ }^{3}$ Si se concibe a la regionalización como la constitución de conjuntos de países

* Investigador del Posgrado en Estudios Internacionales de la Universidad Hankuk de Estudios Extranjeros, Corea del Sur.

Doctor en Relaciones Internacionales por la UNAM reunidos por lazos económicos o políticos; en la historia hay numerosos casos precedentes de espacios políticos y económicos mucho más amplios que los espacios nacionales. A lo largo de varios siglos, la expansión del capitalismo a nivel mundial se ha realizado bajo la forma de imperios coloniales y bloques de cooperación política y económica. ${ }^{4}$

La diferencia entre la regionalización tradicional y la de nuestros días consiste en que la regionalización de la actualidad no implica la constitución de bloques económicos sin dominio político formal. En otras palabras, a diferencia de los bloques anteriores, desiguales y jerarquizados, o sea, esencialmente verticales, los bloques de hoy son más horizontales, es decir, incluyen a países de niveles de desarrollo similares, o de niveles diferentes pero autónomos entre sí. La regionalización en décadas recientes tiene que ver con la extensión o ampliación de las redes de producción en un área geográfica determinada. El surgimiento de estas redes de producción naturalmente ha fortalecido la regionalización: el proceso mediante el cual el comercio y la inversión en el interior de una zona geográfica específica crecen más rápidamente que el comercio y las inversiones del área con el resto del mundo.

En cambio, el regionalismo se refiere a la manera en que se establece la colaboración política a nivel intergubernamental, cuyo principal objetivo es formar la cooperación económica entre los estados participantes. ${ }^{5}$ Sin embargo, en el proceso del progreso de la regionalización hacia el regionalismo se han diferenciado las regiones del mundo. Cada región muestra diferentes niveles de desarrollo de integración económica debido a sus particularidades. La Unión Europea es un bloque políticamente diseñado desde el primer momento y sus pasos posteriores han 
requerido las voluntades políticas de los estados miembros. Hoy en día es el bloque más avanzado y el único que tiene las instituciones políticas comunes, administrativa $\mathrm{y}$ técnicamente hablando. En cambio, la región asiática todavía no tiene una institución formal de integración económica, aunque la economía regional ha alcanzado desde hace tiempo la integración real. En el caso asiático, no ha coincidido la integración real y la formal. En el continente americano se presenta el caso donde la integración real tradicional se ha convertido en la institucional, que a la vez está profundizando aún más la integración real. ${ }^{6}$

\section{La modificación del panorama del regionalismo asiático}

La no coincidencia entre la integración real y la formal, o en otras palabras, el no progreso de la regionalización hacia el regionalismo, en Asia tiene varias explicaciones. En general, los atributos derivados de la historia, la culturas y las ideas diferentes, por ejemplo, crean las particularidades de cada región. Para Asia, el factor más importante que impidió la integración institucional hasta la década de los ochenta fue la experiencia histórica, las diferentes ideologías y los diversos sistemas económicos.

El primer factor del actual estado estático del regionalismo en el noreste asiático tiene que ver con el pasado. Aunque la inversión extranjera directa de Japón en los distintos territorios asiáticos ya contaba con una magnitud considerable, la integración económica formal era difícil de gestar en Asia por la experiencia de la invasión imperialista japonesa en el pasado. Debido a que seguían vigentes en la memoria las actividades imperialistas japonesas, no procedió la integración formal.

Otro factor importante fue la cuestión de la ideología. La presencia del socialismo en China, Corea del Norte y Vietnam ha limitado en cierto sentido el intercambio tanto político como económico con sus vecinos capitalistas hasta los ochenta del siglo XX. Hasta el fin de la guerra fría, el noreste asiático era un símbolo de la cicatriz de la segunda guerra mundial por la división de Corea. La relación entre China y Taiwan también fue otra cuestión a considerar al respecto. ${ }^{7}$

Por otra parte, la gran diferencia de nivel económico ha sido también un obstáculo para la integración económica regional. Es importante poder asegurar los beneficios económicos que se pueden obtener de la integración formal de la región para que ésta se realice. Sin embargo, los distintos niveles de las economías regionales y las diferencias del sistema económico entre países como Hong Kong y Vietnam han sido tan grandes que fue difícil pensar en la integración y esperar los beneficios reales de la integración económica regional.

Sin embargo, se está procediendo desde hace algunos años a la integración regional. La razón por la cual se efectúa ahora el proceso de integración tiene que ver con el cambio del sistema económico de algunos países y el entorno de la economía mundial. A partir de la década de los noventa, casi todos los países del mundo han participado en el movimiento de bloques económicos regionales. Los países de esta región también llegaron a tener interés en la integración económica regional para enfrentar el nuevo entorno económico mundial. ${ }^{8}$ 


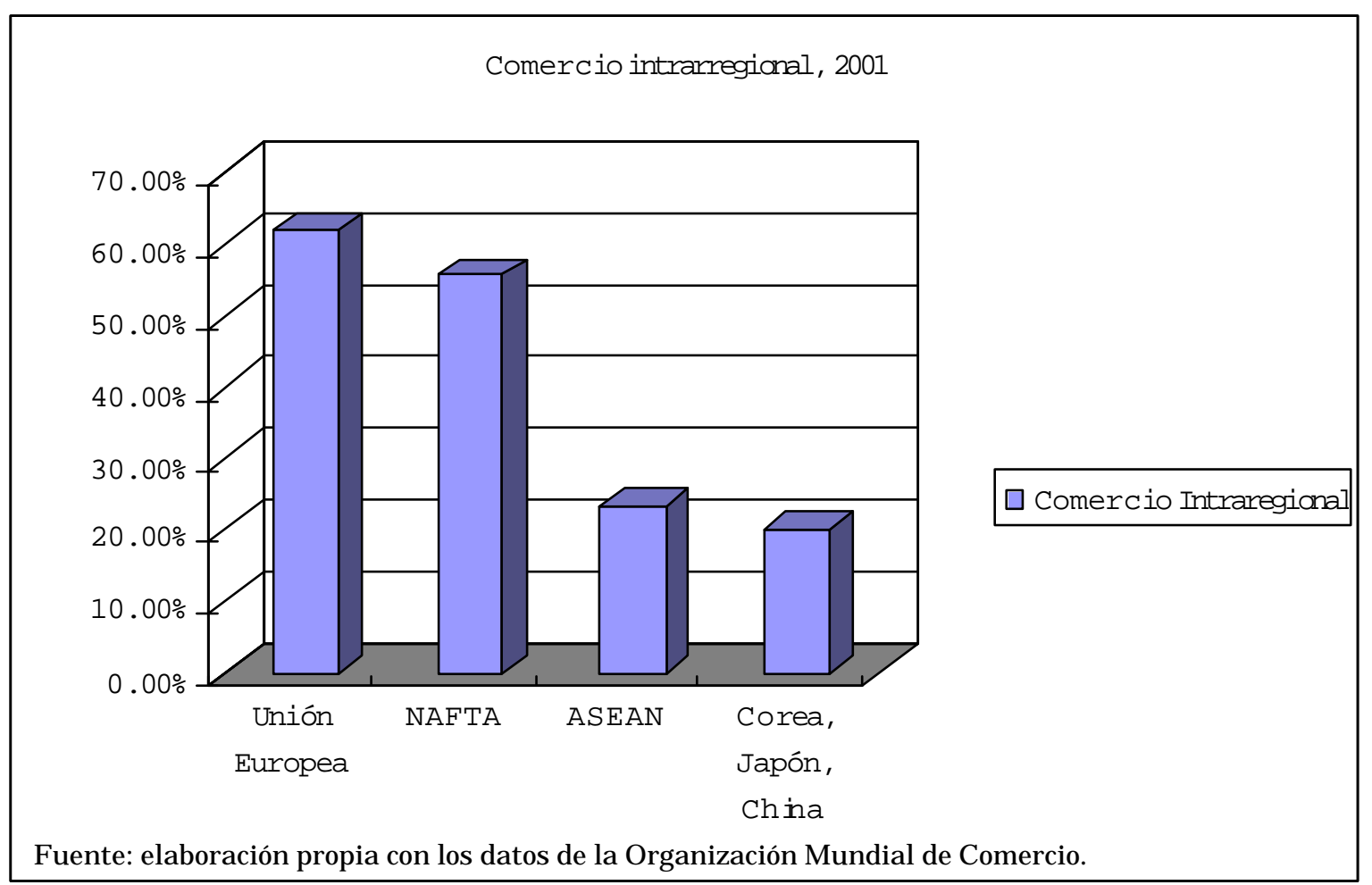

También la apertura económica y la liberalización del comercio e inversión de China hicieron prosperar el intercambio económico regional. En la época en que la cuestión económica es más importante que la ideología, los países regionales consideran la formación del bloque económico regional como una alternativa viable para elevar su potencial.

En esta circunstancia de la economía mundial, se presencia el aumento del comercio intrarregional asiático. La gráfica muestra el porcentaje de este comercio en las principales regiones del mundo.

\section{El comercio intrarregional de las principales regiones económicas}

A diferencia de las otras regiones como Europa y América del Norte, Asia ha mostrado un porcentaje relativamente bajo de comercio intrarregional hasta ahora. Sin embargo, esta tendencia está cambiando, lo que constituye el trasfondo de las posturas de los países regionales. ${ }^{9}$ China ocupó el año pasado el primer lugar en la lista de exportadores para el mercado japonés, con $18.3 \%$ de la importación total de Japón, dejando atrás por primera vez a Estados Unidos, quien registró 17.1\%. Las exportaciones de China siguen creciendo, aun cuando las importaciones totales de Japón cayeron como el año pasado. Asimismo, China surgió el año pasado como el primer mercado importador de las exportaciones coreanas. ${ }^{10}$

El debate actual de la integración económica regional asiática gira en torno al tratado de libre comercio entre Japón, Corea y China. Estos tres países coinciden en la necesidad de la firma del tratado de libre comercio entre sí; sin embargo, muestran posturas diferentes en cuanto a los pasos a seguir. La lógica básica de cada postura consiste en tratar de hacer del nuevo bloque económico regional un foro bajo su influencia, en busca de la hegemonía económica regional. Para Japón el tratado de libre comercio entre los tres países debe ser concertado a la manera de poder defender su competitividad ante el crecimiento repentino de China. Por su parte, China intenta utilizar el tratado para ganar el 
mercado agrario de los otros dos países y recibir el capital y la tecnología de punta.

La iniciativa y el proceso de negociación del TLC entre los tres países centrales del noreste asiático ya tienen varios años de vida. La necesidad de la integración económica regional la sintieron desde finales de la década de los noventa. Y para el primer paso del proceso los tres países pusieron en marcha el Foro de Negocio Corea, Japón y China, acordado en la cumbre de ANSEA+3 en el año $2001 .{ }^{11}$ La primera reunión de dicho foro fue celebrada en noviembre de 2002, en donde se discutieron la necesidad y la factibilidad de crear la comunidad de acero de Corea, Japón y China para la cooperación regional de dicho producto. Esta comunidad sería la precursora de la integración económica regional más profunda, como el caso de la comunidad de carbón y acero de Europa. Sin embargo, las tres naciones mantienen distintas ideas sobre la formación del bloque económico entre ellos.

\section{La posición de China ante la integración económica regional}

China es el país más activo por el momento en la carrera de integración económica regional; su estrategia se muestra claramente en las actividades recientes. En noviembre de 2002 China firmó un acuerdo marco para las negociaciones del tratado de libre comercio con la ANSEA. Cuando se realice esta integración, se creará un mercado único con una población de 1700 millones, cuya producción total sería 1 billón 200 mil millones de dólares. China acordó formar el mercado unificado hasta 2010 con Brunei, Malaisia, Indonesia, Filipinas, Singapur y Tailandia, al tiempo que creará el mismo mercado unificado hasta el año 2015 con Camboya, Laos, Myanmar y Vietnam. ${ }^{12}$ La finalidad de este tratado es elevar la exportación de los productos chinos ganando terreno frente a los japoneses; es decir, la finalidad de China es desplazar la hegemonía económica de Japón en el sudeste asiático para posicionarse como país hegemónico. Para lograrlo, se ayudaría de los comerciantes chinos dispersos en dichos países en donde cuentan con el poder del mercado, por lo cual China actúa rápidamente frente a Japón.

La estrategia de China en su lucha por la hegemonía consiste en formar primero la zona de libre comercio con la ANSEA y luego hacer participar a Japón y a Corea en dicha integración bajo la iniciativa de China. Por otra parte, este país ya propuso de hecho la conformación del área de libre comercio entre Corea, Japón y China. Sin embargo, el procedimiento que propone es la firma de tratados de libre comercio separados entre Corea-China y Japón-China, para que en los dos grandes mercados los productos chinos puedan ganar terreno separadamente, y así poder contrarrestar la influencia de Japón en las negociaciones comerciales y en su potencia económica. ${ }^{13}$

\section{La estrategia de Japón ante el crecimiento de China}

Japón se ha posicionado por muchas décadas como una potencia hegemónica en la economía de Asia. Esta posición vacila ante el crecimiento rápido de China y la depresión prolongada de su economía. El cambio de la postura japonesa frente a la integración económica refleja el cambio de entorno económico tanto interno como externo. Mientras que China se adelantó en la relación económica con la ANSEA, Japón también trata de no perder su potencia e influencia en dicho mercado. Como consecuencia, se firmó un acta de entendimiento para el Tratado de Libre Comercio entre Japón y la ANSEA, en noviembre de 2002. ${ }^{14}$ Se trata de un acuerdo menos desarrollado en cuanto al contenido de la búsqueda de la integración económica regional al compararlo con el de China. Los países de la ANSEA estiman que mediante el acuerdo de libre comercio con Japón las exportaciones incrementarán $50 \%$ y las importaciones desde Japón 25\%. ${ }^{15}$

Mientras que China prefiere las negociaciones paralelas entre los tres países, Japón busca firmar primero el acuerdo de libre comercio con Corea del Sur y luego hacer 
participar a China en dicho esquema de integración. Para Japón el trato directo con China le resulta difícil por la preocupación de la invasión masiva de los productos agrícolas chinos que son sensibles políticamente para Japón. De hecho, Japón y Corea pusieron en marcha primero el APPRI (Acuerdo de Protección y Promoción Recíproca de la Inversión) como etapa previa de la zona de libre comercio. ${ }^{16}$ Por otra parte, Japón está pidiendo a Corea el pronto inicio de las negociaciones comerciales para el acuerdo de libre comercio, concluyendo ahora el estudio conjunto de libre comercio puesto en marcha en 2002 y planeado originalmente con el período de dos años. ${ }^{17}$

Se ve claramente que la intención de Japón es crear una red de rodeo ante el avance chino en la región asiática a través de la alianza con la ANSEA y Corea.

\section{El movimiento de los otros países de la región}

Corea intenta utilizar el papel de intermediario entre Japón y China en busca de su propiobeneficio. Como los otros dos países quieren utilizarla en su juego de hegemonía, Corea analiza el panorama y busca los beneficios del intermediario. La posición actual de Corea es dar prioridad al TLC con Japón, ya que la economía japonesa tiene una estructura industrial semejante a la coreana; además, Corea teme a la competitividad de los productos agrícolas de China.

Singapur, otro país importante en la región, está firmando una amplia red de tratados de libre comercio en su estrategia de supervivencia como un país de comercio de tránsito. Como a Singapur le interesa posicionarse siempre en el centro de la red de finanzas y comercio internacional, le resulta importante utilizar los bloques económicos regionales como instrumento estratégico. Aparte de la puesta en marcha del acuerdo con Japón, Nueva Zelanda y Australia, tiene terminada la negociación con Estados Unidos, y está en negociaciones comerciales con
México, Chile, Canadá y China. A través de la red de tratados de libre comercio, Singapur intenta tener un trámite aduanero simplificado y otras facilidades de comercio que le permitan al país las características necesarias para el comercio en tránsito.

Taiwan también está en negociaciones comerciales para un acuerdo de libre comercio con la ANSEA, al igual que la India.

\section{Comentario final}

$\mathrm{Al}$ igual que en otras regiones de la economía mundial, los acuerdos de libre comercio se han concebido y utilizado en Asia como un instrumento tanto político como económico. Esta región posee las ancestrales herencias singulares de regionalización desde el área de la cultura china antigua hasta el imperio militar japonés de principios del siglo XX. Las particularidades derivadas de la historia y la cultura han hecho que se mantengan características distintas de otras regiones, como la no coincidencia de la integración real con la integración formal en la actualidad.

La hegemonía económica que ha logrado Japón en la región, actualmente enfrenta el serio reto de China. La característica de esta carrera es que los rivales intentan utilizar la integración económica regional como un instrumento para contrarrestar al otro lado. En este proceso, las distintas modalidades de búsqueda de la integración regional por parte de China y Japón muestran claramente el carácter político y económico del bloque económico regional. Lo importante en las negociaciones del acuerdo de libre comercio es el costo y el beneficio para el contratante. Las ganancias y las pérdidas económicas por la liberalización de comercio e inversión serán las prioridades a considerar en la búsqueda de la integración regional. Debido a que el comercio intrarregional continúa creciendo, China y Japón intentarán concretar continuamente los distintos tratados de libre comercio. Sin embargo, el panorama de las redes que prefieren los dos países continuará siendo distinto bajo la lógica de la búsqueda 
de la hegemonía en la región. En el noreste asiático, el tratado de libre comercio está siendo utilizado para afrontar la creciente rivalidad de la hegemonía económica de la región.

\section{Notas}

1 Jeffrey Frankel A., Regional Trading Blocs in the World Economic System. Institute for International Economics, p. 4, Washington DC, 1997.

Aunque el APEC es el nuevo intento de regionalismo abierto y se podría definir como el tratado económico en vez de tratado comercial, aquí se cita la expresión original usada por el autor Jeffrey Frankel.

2 Jaime de Melo y Arvind Panagariya dicen que se llaman olas porque llegaron de repente. La primera ola llegó en los años cinco y sesenta, mientras que los nuevos acuerdos comerciales regionales, la segunda ola, se extienden por todo el mundo a partir de los noventa. Jaime de Melo y Arvind Panagariya, New Dimensions in Regional Integration, Cambridge University Press, 1993, p. 3,.

3 La globalización y la regionalización están interrelacionados. Los procesos de regionalización económica son consecuencia de la globalización y, a la vez, la facilitan al unir los mercados nacionales en las latitudes determinadas debido a su propia lógica de la integración económica regional, como la necesidad de expansión y reproducción del capital a escala mundial. Los dos procesos complementarios, globalización y regionalización, no son fenómenos nuevos en cierto sentido; sus manifestaciones datan de varios siglos atrás. Véase Aldo Ferrer, Historia de la globalización, México, FCE, 1996.

4 Faysal Yachir, Bloques regionales en la economía mundial, México, pp. 91-93, vol. 26, núm. 103, octubrediciembre, 1995.

5 María Cristina Rosas, "Globalización y regionalismo: ¿procesos antagónicos o complementarios?”, Relaciones Internacionales, p. 28, núm. 71, julio-septiembre, 1996; John Ravenhill, "Competing Logics of Regionalism in the Asia-Pacific" en Révue d'integration européenne, Canadá, XVIII, núms. 2-3, 1995, p. 179.

6 Faysal Yachir, op. cit., pp. 94-97.

7 Nam Kwon Mun, "El impacto del TLCAN en las relaciones económicas entre Corea del Sur y México", tesis doctoral, pp. 22-23, UNAM, 2002.

8 Con respecto al florecimiento de la integración económica en los noventa, Baldwin presenta la teoría del efecto dominó. Su argumento está basado en el hecho de que los países de fuera del bloque perciben los beneficios de la membresía, y llegan a solicitar el ingreso al bloque o establecer entre ellos un nuevo bloque económico. En otras palabras, la fuerza que impulsa la unión y su tendencia al regionalismo es, irónicamente, la de los efectos adversos de no pertenecer a la integración económica.

Richard E. Baldwin, “A Domino Theory of Regionalism”, en Trading Blocs, MIT, 1999, pp. 479-500.
9 Con respecto a la razón del relativamente bajo intercambio económico regional de Asia, se muestra la presencia de Estados Unidos en el comercio de estos países, y también los distintos sistemas y niveles económicos de los países.

10 Los datos citados son de la Secretaría de Hacienda de Japón y la Asociación Coreana de Comercio.

11 La cumbre ANSEA+3 es una conferencia anual donde se reúnen, aparte de los presidentes de los países de la ANSEA, los presidentes de Corea, Japón y China. Los estadistas de los tres países acordaron establecer el Foro de Negocio Corea, Japón y China en dicha cumbre de 2001, con el objetivo de discutir el tratado de libre comercio entre los tres países. La Asociación de Naciones del Sudeste Asiático es un foro de cooperación político y económico entre los once países. Se creó el 8 de agosto de 1967, y se ha ampliado su pertenencia.

12 Reportaje del periódico Chosun, el día 5 de noviembre de 2002, www.chosun.com.

13 El 5 de noviembre el primer ministro chino habló de su intención de realizar un tratado de libre comercio con Japón y Corea. Pero en la metodología del procedimiento hizo hincapié en el proceso paralelo de negociaciones con Corea y Japón.

14 Japón firmó el acuerdo con la ANSEA el 5 de noviembre de 2002 en Phnom Penh, Camboya.

15 Reportaje del periódico Chosun, el día 5 de noviembre de 2002, www.chosun.com.

16 El APPRI entre Corea y Japón empezó su negociación en noviembre de 1998, y se firmó el 2 de diciembre de 2002. La entrada en vigor se efectuó el 1 de enero de 2003.

17 El estudio conjunto entre Corea y Japón tenía planeado un período de dos años. Todavía Corea no tiene una postura determinada ante la solicitud de Japón para iniciar las negociaciones de tratado, ya que no está seguro de los efectos económicos.

El instituto público encargado del estudio estima una mejora de balanza de pagos por unos 9000 millones de dólares, puesto que se bajará el precio de los componentes japoneses y se aumentarán las exportaciones coreanas.

\section{Bibliografía}

Baldwin, Richard E., "A Domino Theory of Regionalism”, en Bhagwati et al. Trading Blocs, MIT, 1999.

CEPAL, El regionalismo abierto en América Latina y el Caribe, 1994.

De Melo, Jaime y Arvind Panagariya, New Dimensions in Regional Integration, Cambridge University Press, 1993. Ferrer, Aldo, Historia de la globalización, FCE, 1996.

Frankel, A. Jeffrey, Regional Trading Blocs in the World Economic System, Institute for Internacional Economics, Washington, D.C., 1997.

Hong Yoo Soo et al., El panorama de cooperación Corea, China y Japón en la industria de tecnología de información y la estrategia posterior (texto en coreano), KIEP, 2002.

Kim Yang Hee et al., La influencia del TLC entre Corea y Japón en la inversión extranjera directa y su significado político (texto en coreano), KIEP, 2001. 
Krasner, Stephen D., "Bloques económicos regionales y el fin de la guerra fría”, en Gustavo Vega CanovasLiberación económica y libre comercio en América del Norte, El Colegio de México, 1993.

Krippendorff, Ekkehart, El sistema internacional como historia, FCE, 1993.

Krugman, Paul, "Regionalism versus Multilateralism: Analytical notes", en Bhagwati et al., Trading Blocs, alternative approaches to analyzing preferential trade agreements, The MIT Press, USA, 1999.

Lawrence, Robert Z., Regionalism, Multilateralism, and Deeper Integration, The Brookings Institution, 1996.

Park Sung-Hoon, (texto en coreano), [La investigación sobre la armonía entre el regionalismo abierto del APEC y la OMC], KIEP, 1998.

\section{Sitios web consultados}

La Organización Mundial de Comercio: www.wto.org

El Diaro Chosun: www.chosun.com

El Diario Hankyoreh: www.hani.co.kr 